\title{
1 Reinforcement meta-learning optimizes visuomotor learning
}

2 Taisei Sugiyama ${ }^{1}$, Nicolas Schweighofer ${ }^{2}$, Jun Izawa ${ }^{3 *}$

3

$4 \quad{ }^{1}$ Empowerment Informatics, University of Tsukuba, Tsukuba, Ibaraki, 305-8573, Japan

$5 \quad{ }^{2}$ Biokinesiology and Physical Therapy, University of Southern California, Los Angeles, CA,

$6 \quad 90089-9006$ USA

$7 \quad{ }^{3}$ Engineering, Information, and Systems, University of Tsukuba, Ibaraki 305-8573, Japan

8

$9 \quad *$ Corresponding author:

10 Jun Izawa

11 Faculty of Engineering, Information, and Systems

12 University of Tsukuba

13 1-1-1 Tennodai, Tsukuba, Ibaraki 305-8573, Japan

14 Tel.: +81-29-853-3756; Email: izawa@emp.tsukuba.ac.jp

15 


\section{Abstract}

17 Reinforcement learning enables the brain to learn optimal action selection, such as go or not go,

18 by forming state-action and action-outcome associations. Does this mechanism also optimize the

19 brain's willingness to learn, such as learn or not learn? Learning to learn by rewards, i.e.,

20 reinforcement meta-learning, is a crucial mechanism for machines to develop flexibility in

21 learning, which is also considered in the brain without empirical examinations. Here, we show

22 that humans learn to learn or not learn to maximize rewards in visuomotor learning tasks. We also

23 show that this regulation of learning is not a motivational bias but is a result of an instrumental,

24 active process, which takes into account the learning-outcome structure. Our results thus

25 demonstrate the existence of reinforcement meta-learning in the human brain. Because motor

26 learning is a process of minimizing sensory errors, our findings uncover an essential mechanism

27 of interaction between reward and error.

30 Keywords: meta-learning, reinforcement learning, motor learning, cerebellum, basal ganglia,

31 reward, punishment, saving

Learning is considered a skill that can be improved by experience and motivation ${ }^{1,2}$. In fact, animals and humans often exhibit accelerated learning over training sessions ${ }^{3}$. How does the brain learn to learn quickly in variable tasks and environments?

Accelerated learning has often been reported in motor learning tasks where human participants learn to compensate for force or visual perturbations to generate a planned movement trajectory ${ }^{4-8}$. After motor memories formed in the initial learning session have been washed out,

40 learning in the second learning session becomes faster than learning in the first session, a 41 phenomenon that is known as the 'saving effect' ${ }^{4,9}$. In this task, motor learning is considered a 
42 process of minimizing sensory prediction errors, i.e., the discrepancy between the generated and 43 predicted movement trajectories is independent of the motivational signals ${ }^{10-12}$. Research has 44 shown that this saving effect is achieved by updating the learning rate (i.e., the policy of how 45 much the motor memory is updated in response to the perceived sensory errors), which is driven 46 by prior experience of the errors ${ }^{7}$. Additionally, recent studies suggest that there is a significant 47 effect of motivational signals on the learning speed ${ }^{13-17}$. How does the brain incorporate motivational signals into the history of errors to regulate learning rates?

One suggested mechanism is a passive, Pavlovian (cue-outcome-based) process in which the valence (reward or punishment) biases the learning rates. For example, in the go and no-go learning task, valence biases the speeds as well as the asymptotes of learning curves ${ }^{18}$. In saccadic adaptation, rewards increase the speeds of adaptation, which is induced by dopaminergic modulation of error signals ${ }^{19}$. While this motivational, hard-wired process might induce biases in error sensitivity of motor learning, it might not explain variations in reward influence of motor learning: some studies show that learning is facilitated by motivational signals ${ }^{14}$, while others report no effect ${ }^{16,17}$, or even decelerated learning ${ }^{15}$. Thus, these variations imply that another mechanism other than the hard-wired mechanism regulates the speed of learning.

In theory, the ultimate goal of motor learning is to maximize future rewards ${ }^{20}$. Thus, reinforcement learning, which is driven only by reward feedback without any sensory prediction 60 error, also forms motor memory ${ }^{21-23}$. Because the spatial generalization function of learned memory, which reflects properties of neural basis of adaptation, is significantly different for errorbased and reward-based learning, two dissociable neural mechanisms are likely involved in errorand reward-based motor learning ${ }^{24}$. reinforces motor learning, which we call 'reinforcement meta-learning.' In contrast to a passive 
process, this learning process is active and instrumental (action-outcome-based), where a higherlevel reinforcement learning mechanism trains a lower-level motor learning mechanism. learning where the parameters characterizing learning behaviors such as learning rates are modulated by high-order reinforcement learning ${ }^{25,26}$. This idea can account for changes in learning rates in decision-making tasks in animals and humans ${ }^{27}$. However, the existence of reinforcement meta-learning in the brain has been difficult to verify experimentally because in decision-making tasks, both learning and meta-learning are updated by a reward feedback, obscuring the contribution of each learning layer.

Here, we devise a reinforcement meta-learning task in which the feedback for learning and that for meta-learning are dissociated: sensory error feedback is provided in a motor learning trial, and reward feedback that depends on the rate of motor learning is provided in a subsequent meta-learning trial. If the brain employs reinforcement meta-learning, it integrates sensory error feedback and reward feedback to form associations between these two to regulate how large motor commands should be updated in response to the observed sensory error. Thus, manipulating the relationship between the extent of learning and the reward feedback should influence how motor learning rates are modulated as a result of meta-learning regardless of which reward and punishment are presented. Alternatively, if the modulation of learning rate is hard-wired to valence, such manipulation might not influence learning rates.

\section{Results}

Forty one healthy participants gave informed consent before participating in the experiment, which was approved by the Institutional Review Board at the University of Tsukuba. One subject was excluded from the analysis since he reported an explicit strategy to perform task. The participants sat on a chair, held the handle of a robot manipulandum with their right arm, and made shooting-like quick movements by displacing the handle of the robot on a horizontal plane ${ }^{24}$. 
92 A computer projector displayed all visual stimuli onto a flat opaque board that occluded both the

93 manipulandum and the arm. The visuomotor meta-learning paradigm was composed of a sensory-

94 error (S) trial and a monetary feedback (M) trial. In the S trial, the visual cursor was projected on

95 the screen, which provides online feedback of the hand (Figure 1A, left). This cursor was rotated

$96 \pm 7^{\circ}$ with respect to the hand movement to induce a sensory prediction error, i.e., the error between

97 the predicted and generated movements. The goal for the shooting was presented with an arc of

$98 \pm 45^{\circ}$ instead of a target, and the participants were asked to randomly choose a movement direction

99 and cross somewhere on the arc except the edge, which emphasizes the sensory prediction error

100 and minimizes the extent of how reach error interferes with task performance. This $\mathrm{S}$ trial was

101 followed by $\mathrm{M}$ trials, in which the small visual target, instead of the arc, was presented, and the

102 participants were asked to shoot at the center of the target as accurately as possible without the

103 cursor feedback. After the shooting movement, the participants received monetary feedback,

104 which was presented as a numerical score (Figure 1A, right). This score was computed as a

105 function of the size of the memory update (learning), which was measured by the aftereffect (i.e.,

106 the changes in the reach direction following the previous sensory prediction error). The

107 participants repeated the cycles of one $\mathrm{S}$ trial followed by four M trials.

This task structure implements a reinforcement learning problem among trial sequences

109 formalized as a Markov decision process, whereby the agent observes the environmental state,

110 responds according to its policy and is rewarded for the action ${ }^{28}$. In our task, observation of the sensory prediction error (state observation) and the learning rate (policy) yielded a memory update

112 (action), which subsequently generated a monetary feedback (outcome, Figure 1A, bottom). Thus,

113 if the brain learns to maximize the monetary feedback, the action-outcome structure (i.e., how

114 much is gained by how much is learned) should determine the change in learning rate. In addition,

115 if the effect of this action-outcome structure is dominant, the valance which has been considered

116 to influence learning rates ${ }^{13}$ does not influence the change in learning rate. 
119 followed by a generalization phase. The training phase had 28 cycles, each containing one $\mathrm{S}$ trial 120 and four $\mathrm{M}$ trials. The generalization phase included four short sequences of visuomotor rotation 121 trials to assay the generalizability of meta-learned learning rates to a conventional visuomotor learning task (Figure 1B).

An example of the visual rotation pattern $\left(+7^{\circ}\right.$ after $\left.-7^{\circ}\right)$ is shown in Figure 1C. In the first (baseline) block, no score was given in $M$ trials. Nevertheless, after the sensory prediction error led by visual cursor rotation was observed at the $k^{\text {th }}$ trial ( $\mathrm{S}$ trial), the reach direction was updated to compensate for the given rotation. Thus, the change in movement direction at the $k^{\text {th }}+$ 1 trial was significantly different from that at the $k^{\text {th }}-1$ trial (cyan arrow in Figure 1C, Wilcoxon paired signed-rank tests, $V=726, p<0.00001, r=0.70$ ), indicating that the robust aftereffects of memory updates were induced by the sensory prediction errors, which is congruent with previous reports of the roles of sensory prediction errors on updates of motor memory ${ }^{29}$.

$132=10$ /group) with different action-outcome structures and valences. In the learn (Lrn) structure, 133 larger aftereffects yielded larger scores, whereas in the not-learn (NLrn) structure, smaller 134 aftereffects yielded larger scores, indicated by the background gradation colors in Figure 1D. The 135 valence determined whether the monetary feedback was positive (reward [Rwd]) or negative 136 (punishment [Pun]). This design required the participants to either learn more to gain more rewards (Lrn-Rwd) and avoid larger punishments (Lrn-Pun) or learn less to gain more rewards 138 (NLrn-Rwd) and avoid larger punishments (NLrn-Pun). To visually observe differences in the 139 regulation of memory updates among these conditions, we focused on the same example rotation 140 pattern $\left(+7^{\circ}\right.$ after $\left.-7^{\circ}\right)$ as that for the baseline analysis (Figure 1D), and the same Wilcoxon paired 141 signed-rank test was performed. The analysis showed robust aftereffects in the Lrn groups but not in the NLrn groups (Lrn-Rwd: $V=53, p=0.0059, r=0.80$; Lrn-Pun: $V=55, p=0.002, r=0.91$; NLrn-Rwd: $V=37, p=0.38, r=0.10$; NLrn-Pun: $V=18, p=0.38, r=0.10)$. That is, when the 
memory update led to an increase in rewards and a decrease in punishment, the aftereffect was kept robust; in contrast, when the memory update led to a decrease in rewards and an increase in punishment, the aftereffect was attenuated.

We considered that the participants regulated the learning rate in accordance with the action-outcome structure (Lrn v.s. NLrn). To confirm this, for all combinations of perturbations $\left(+7^{\circ}\right.$ after $+7^{\circ},-7^{\circ}$ after $-7^{\circ},+7^{\circ}$ after $-7^{\circ}$, and $+7^{\circ}$ after $\left.-7^{\circ}\right)$, we estimated the learning rate, $\beta$, by taking the ratio of the memory update (aftereffect) to the sensory prediction error. In other words, we computed how much the reach direction changed relative to the sensory prediction error in an $\mathrm{S}$ trial (see Methods for details). The median $\beta$ was taken for each individual participant and each block for analysis. Figure $2 \mathrm{~A}$ shows the group mean of individual medians over the blocks for each group and illustrates that the increase or decrease in learning rate over the blocks was different for the groups. To confirm this, we examined the trend of the learning rate over the blocks using a linear mixed-effect model with the action-outcome structure, valence, and block as the fixed effects and participant as the random intercept effect (see Methods for details). This analysis revealed a significant interaction between the action-outcome structure and the block $(t$ $\left.=-2.65, p=0.009, R^{2}=0.61\right)$. Additionally, we estimated marginal slopes for the change in learning rate over the blocks with respect to the action-outcome structure, which confirmed that the change in learning rate over the blocks was larger in the Lrn groups than in the NLrn groups $(t=-2.61, p=0.0099)$. However, we did not find a significant effect of valence $(t=1.76, p=$ 0.08). Furthermore, we computed the change in learning rate compared with the baseline for each block and then took the individual participant means of this value across the blocks ( $\Delta \beta$, Figure 2B). This result was then analyzed by a reduced linear model with the action-outcome structure and valence as the fixed effects (see Methods for details). Then, we confirmed that the change in

167 learning rate $(\Delta \beta)$ was larger in the Lrn group than in the NLrn group $(t=-2.14, p=0.04$, $\left.168 R^{2}=0.15\right)$, while neither valence $\left(t=1.21, p=0.23, R^{2}=0.15\right)$ nor the interaction between action- 
169 outcome and valence $\left(t=-0.38, p=0.70, R^{2}=0.15\right)$ has a significant effect. These results

170 demonstrate that the participants regulated their learning rates according to the action-outcome

171 structure regardless of valence.

If these regulations are achieved by reinforcement learning of learning rates, outcomes that were provided as scores should be maximized. Figure $2 \mathrm{C}$ shows the across-subjects mean of the block total score in all conditions. Notably, the baseline was not presented to the participants but was calculated in the same way as the following intervention blocks. As a result of the regulation of the learning rate, participants in all conditions increased their monetary outcome (one-sample Wilcoxon-test; Lrn-Rwd: $V=48, p=0.04, r=0.56$, Lrn-Pun: $V=49, p=0.03, r=0.61$ NLrn-Rwd: $V=55, p=0.002, r=0.91$, NLrn-Pun: $V=49, p=0.03, r=0.61)$. These results support the hypothesis that the participants both up- and down-regulated the learning speeds via reinforcement meta-learning: instrumental and active meta-learning processes. something else specific for this training task? Subsequently, after the training phase, the participants experienced the generalization phase, which involved a conventional visuomotor rotation task in which both the visual target and the hand cursor were presented and no monetary feedback was provided (Figure 1B). Then, we investigated the learning rate in the generalization phase to determine whether the effect of the action-outcome structure in the reinforcement metalearning was transferred to a conventional visuomotor rotation task. Additionally, we investigated time-dependent changes within the generalization phase to determine the temporal robustness of the effect. To do so, we estimated the learning rate, $\beta$, for each individual, block, and sequence, and then the individual mean change from baseline across the blocks, $\Delta \beta$, was calculated for each sequence. Figure 3A shows the group means for individual mean change over the sequences for the Lrn and NLrn groups. The individual mean change in learning rate was analyzed with a linear mixed-effect model with the action-outcome structure, the sequence, their interaction as 
194 fixed effects and the participant as the random intercept effect (see Methods for details). The analysis revealed a significant effect of the action-outcome structure $\left(t=-2.08, p=0.04, R^{2}=0.67\right)$ and its interaction with the sequence $\left(t=2.58, p=0.01, R^{2}=0.67\right)$. Furthermore, the individual mean changes in learning rate for the training phase correlated with those in the first half (Figure $\left.3 \mathrm{~B}, R=0.43, t_{38}=2.90, p=0.006\right)$ but not with those in the second half $\left(R=0.25, t_{38}=1.61, p=\right.$ 0.12) of the generalization phase. Notably, although the change in learning rate was greater in the Lrn groups than in the NLrn groups in the first half (vertical axis of Figure $3 \mathrm{~B}, t=-2.78, p=$ $0.008, d=.88)$, this phenomenon was not observed for the second half $(t=-0.74, p=0.46, d$ $=.23$ ). These results demonstrate that the regulation of the learning rate by reinforcement metalearning in the training phase was generalized to the learning rate in motor learning for conventional visuomotor rotation tasks. This suggests that what is updated in the training phase overlaps with the learning rate of visuomotor learning.

\section{Discussion}

We found that by presenting rewards as a function of the amount of memory update that was induced by sensory prediction errors, the brain both up- and down-regulated the learning rates to increase rewards as well as to avoid punishment. This effect was gradually developed over the training sessions, and the directionality of regulation (i.e., learn or not-learn) was determined by the action-outcome structure, not by valence. These results suggest that reinforcement learning is employed to regulate learning rates. Furthermore, by examining the generalization of these regulations from the training task to the conventional visuomotor rotation task, we found that these meta-learning effects were transferred to motor learning. This observation indicates that there is an overlapping neural basis between reinforcement metalearning and visuomotor learning. Thus, our results demonstrate the existence of a reinforcement meta-learning mechanism for motor learning in the human brain.

In machine learning studies, automatic tuning of learning parameters has been a long- 
219 standing problem ${ }^{30}$. A computational model of biological reinforcement learning suggests that, in

220 the brain, neuromodulators such as noradrenaline, acetylcholine, and noradrenaline adjust

221 learning parameters of reinforcement learning ${ }^{31}$ via reward-based modulation of these

222 parameters ${ }^{32}$. Recent algorithms of meta-learning in machine learning studies have highlighted a

223 hierarchical structure composed of two reinforcement learning systems: while a low-order

224 reinforcement learning optimizes the weight parameters for action selections in a single learning

225 episode, a high-order reinforcement learning optimizes meta-parameters of the low-order reinforcement learning network to maximize rewards across multiple learning episodes ${ }^{25,26}$.

227 Although the neural implementation of such reinforcement meta-learning was recently 228 discussed $^{27}$, there was no experimental examination. Here, we devised a meta-learning paradigm

229 in which reward feedback for meta-learning, which was provided independently from the sensory

230 error feedback for motor learning, was manipulated as a function of the learning rate of motor

231 learning. Our data demonstrated that while motor memory was updated to minimize the given sensory prediction errors in a single trial, the learning parameter of motor learning was updated to maximize rewards over multiple trials.

The observed meta-learning effect may account for previous reports of learning-rate

235 flexibility during motor learning tasks ${ }^{6,7,13,33}$. For instance, the learning rate increases over the

236 sessions when the perturbation is relatively constant but decreases when the direction of 237 perturbation frequently changes. Importantly, a typical learning paradigm with constant perturbation shares the same action-outcome structure as that for Lrn-Pun, whereby a memory update attenuates movement errors on the next trial, which can be considered avoidance of aversive outcomes ${ }^{34,35}$. In this case, reinforcement meta-learning accelerates learning rates to

241 quickly reduce errors in re-learning (e.g., saving) ${ }^{6}$ or learning with additional punishment 242 signals ${ }^{13}$. Conversely, a non-typical learning paradigm with rapidly changing perturbation shares 243 the same action-outcome structure as NLrn-Pun because the memory update increases errors in 244 the next trial. In this case, reinforcement meta-learning decreases the learning rate. Thus, 
reinforcement meta-learning explains how the statistics of the perturbation affect learning rates ${ }^{7,33}$.

According to a conventional theory of motor learning, the brain updates motor

247 commands independent of reward to minimize sensory prediction errors ${ }^{36}$. Although motor

248 memory has also recently been found to be updated by rewards, the neural basis of this reward-

249 based motor learning is likely distinct from that of sensory error-based motor learning ${ }^{24,37}$.

250 According to these previous studies, rewards might not interact with sensory prediction errors

251 during motor learning. Here, our data demonstrate that the sensory prediction errors and the

252 rewards presented in separated trials were integrated at the higher-level motor learning system,

253 i.e., meta-learning, to regulate learning rates.

In theory, to establish reinforcement meta-learning for motor learning, the sensitivity to sensory prediction errors should be evaluated by the rewards. Thus, our results suggest a close interaction between rewards and sensory prediction errors during motor learning. Because research evidence suggests the involvement of cortico-basal ganglia networks in reinforcement meta-learning ${ }^{27}$ and the cerebellum in sensory error-based learning ${ }^{38,39}$, reinforcement meta-

259 learning for motor learning is likely mediated by the functional connectivity between these two

260 learning systems. Thus, the anatomical projections between the basal ganglia and the cerebellum

261 could have a computational role in reinforcement meta-learning ${ }^{40}$. This possibility is further supported by recently reported reward-related signals in cerebellar inputs ${ }^{41}$ and outputs ${ }^{42}$ during motor control tasks. We suggest that these interactions between the basal ganglia and the cerebellum play a key role in optimizing learning parameters for motor learning via reinforcement

265 learning.

\section{Acknowledgments}

268 This work was supported by KAKENHI (grant numbers 17H06023 and 19H04977). T.S. is 269 grateful for financial support from the JSPS Research Fellowship for Young Scientist and 


\section{Author Contributions}

273 Conceptualization, T.S. and J.I.; methodology, T.S. and J.I.; investigation, T.S. and J.I.; formal

274 analysis, T.S. and N.S.; writing - original draft, T.S., and J.I.; writing - review \& editing, T.S.,

275 N.S., and J.I.; funding acquisition, J.I.

276

277 Declaration of Interests

278 The authors declare that they have no competing interests. 
A Sensory-error feedback trial
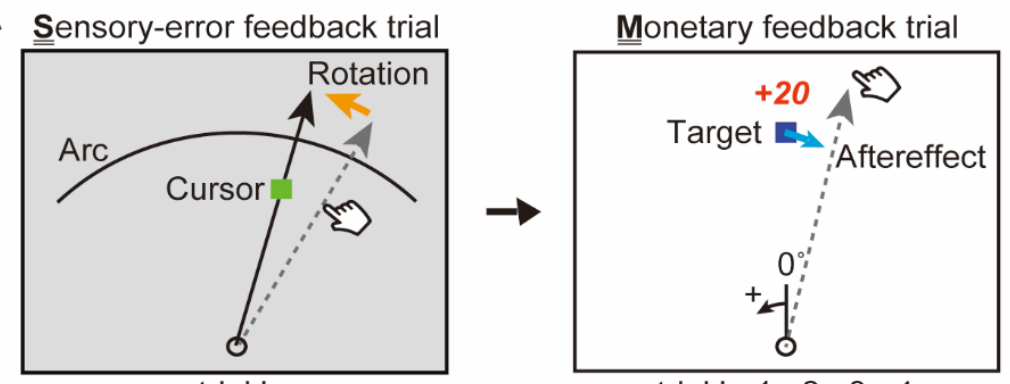

trial $\mathrm{k}$

trial $\mathrm{k}+1,+2,+3,+4$
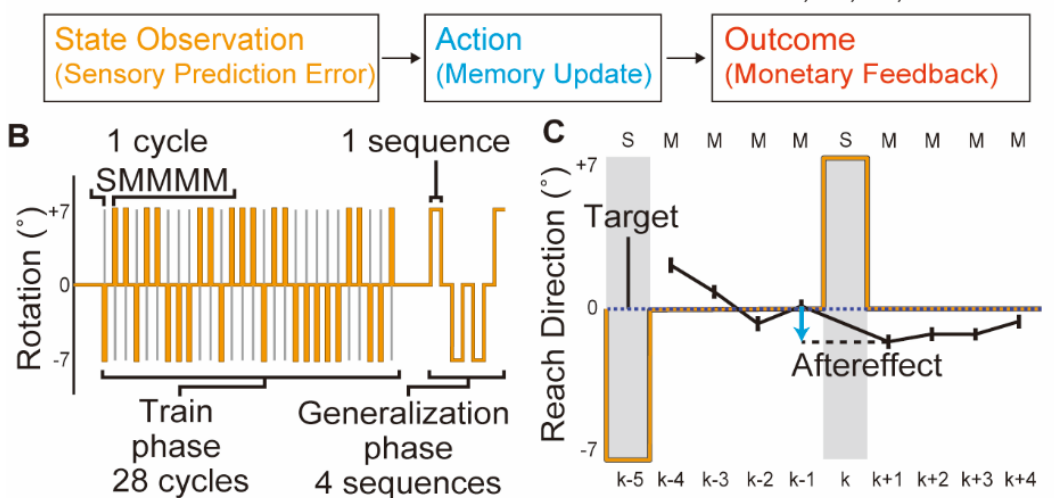

D Reward (Rwd) Punishment (Pun) $+20 \square-20$
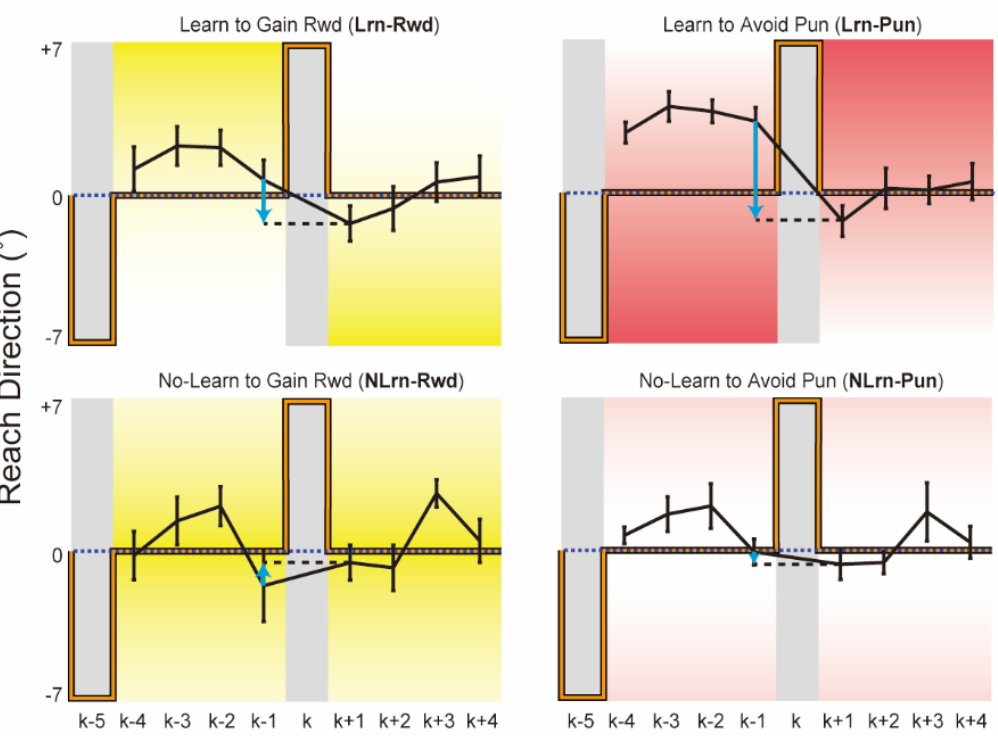

280 Figure 1. Reward-based visuomotor meta-learning paradigm. (A) Training paradigm. Cursor rotation (orange arrow)-induced sensory prediction error observation (state) in the sensory error

282 (S) trial. The aftereffect size (action, cyan arrow) was scored (outcome) in the monetary feedback 283 (M) trial. (B) One block consisted of the training phase, which included 28 cycles of $1 \mathrm{~S}$ trial 
284 followed by $4 \mathrm{M}$ trials. After brief washout (null) trials, the generalization phase was composed

285 of four short sequences of visual rotation trials without scores. (C) Plot of reach direction w.r.t.

286 the target direction (memory) for one example rotation pattern in baseline. The aftereffect (cyan

287 arrow) was developed in the opposite direction to the rotation (orange line) in the $\mathrm{S}\left(k^{\text {th }}\right)$ trial,

288 showing a motor memory update. (D) Memory profile for the same rotation pattern for each group.

289 The scores improved with memory updates toward the yellow zone (large reward) or away from

290 the red zone (large punishment). The error bars indicate standard error of the mean (SEM). 

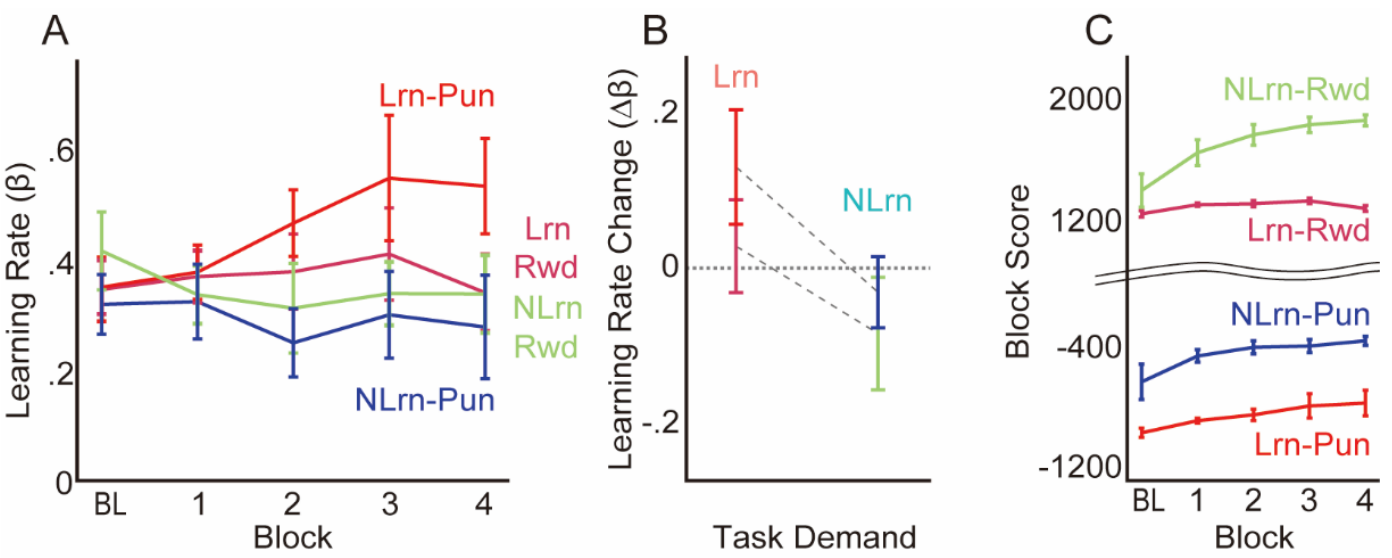

293 Figure 2. Mean performance scores and learning rates. (A) Estimated changes in learning rates by block. (B) The learning rates changed more in the Lrn group than in the NLrn group. The error bars indicate SEM. (C) Total scores for each block. The baseline (BL) scores were calculated and are shown here. 

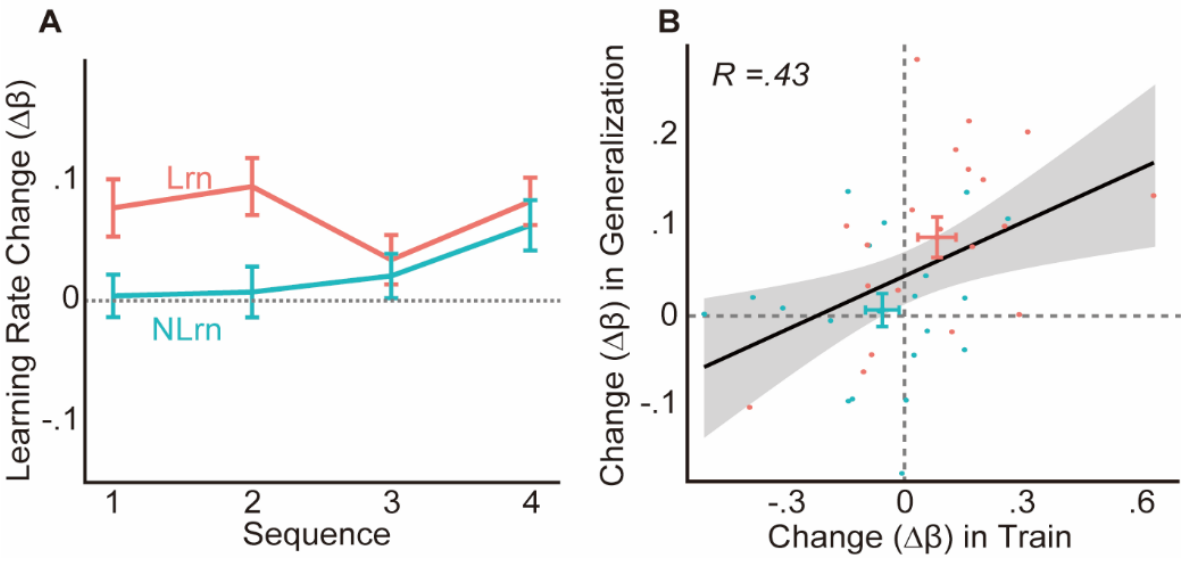

299

Figure 3. Mean learning-rate changes in the generalization phase. (A) Mean learning-rate

301 changes across the blocks for each sequence. (B) The learning-rate changes in the training phase

302 corresponded with those in the first half of the generalization phase. Each dot represents the data

303 from an individual participant. The error bars indicate SEM. 


\section{Participants}

306 Forty one right-handed participants (25 males; aged 19-37 years, $\mu=24$ ) volunteered for this

307 experiment. Their handedness was confirmed by the Edinburgh Handedness Inventory, and they 308 reported no history of neurological or motor disorders. We excluded one subject who reported an 309 explicit strategy to perform the task from the analysis. They were paid 1,640 JPY for their 310 participation, with an additional performance-based compensation up to 1,000 JPY.

\section{Task design}

\section{General}

313 Participants performed the task using a robot manipulandum ${ }^{24}$ that moved only in the horizontal

314 plane. They sat on a chair and held the robot handle in their right hand. A horizontal, flat, opaque

315 board covered the task space, occluding the hand and forearm. A computer projector was fixed 316 above and displayed visual information on the board.

317 In each trial, participants made a rapid shooting movement when a target appeared $10 \mathrm{~cm}$ away

318 from the starting point. To control for use-dependent learning ${ }^{43}$, the target location was pseudo-

319 randomly selected from 1 of 7 locations: $-15^{\circ},-10^{\circ},-5^{\circ}, 0^{\circ}$ (directly in front of the participant),

$3205^{\circ}, 10^{\circ}$, and $15^{\circ}$. The counter-clockwise direction was defined as positive in the angular 321 coordinates (Figure 1A). To maintain similar kinematics across trials, "Too Fast" or "Too Slow" 322 was displayed as a warning when the movement duration was $<200 \mathrm{~ms}$ or $>300 \mathrm{~ms}$, respectively. 
324 There were four trial types: Null, Sensory-error feedback (S), Monetary feedback (M), and

325 Generalization. In Null trials, participants made shooting movements toward the targets with

326 veridical online cursor feedback. In S trials, the targets were replaced with an arc spanning $\pm 45^{\circ}$,

327 centered on the home position and with $10 \mathrm{~cm}$ radius (Figure 1A) ${ }^{44}$. Participants were asked to

328 cross the arc while trying to distribute their reach direction across $\mathrm{S}$ trials. Online cursor feedback

329 was rotated $\pm 7^{\circ}$ from hand movements to induce errors between the predicted hand position and

330 the visual feedback. The rotation direction was pseudo-randomly selected (Figure 1B). Note that

331 the use of an arc instead of a target minimizes the extent of how reach errors interfere with task

332 performance and, thus, emphasizes errors between the observed and predicted hand movements,

333 i.e., sensory predictor errors. In M trials, participants made shooting movements toward targets

334 without online cursor feedback. Upon movement completion, monetary feedback was presented

335 as a numerical score above the target (Figure 1A). Scores were computed based on the reach

336 direction from the target, with different computations across the experimental conditions (see

337 below). Finally, Generalization trials were identical to Null trials except that, similar to S trials,

338 online cursor feedback was rotated $\pm 7^{\circ}$ from the hand movement, as in conventional visuomotor

339 rotation tasks ${ }^{45}$.

340 Participants performed first one Baseline block, in which no monetary feedback was provided,

341 and then four blocks with monetary feedback. Each block comprised a Train phase with 28

342 cycles of $1 \mathrm{~S}$ trial and $4 \mathrm{M}$ trials, followed by a Generalization phase. Brief washout blocks of 14

343 Null trials were inserted before each phase. The Generalization phase comprised 4 Sequences of

3445 consecutive cursor rotation trials, with 5 Null trials between each Sequence (Figure 1B). The

345 aim of the Generalization phase was to test whether the participants generalized the meta-learning

346 effect formed in the Train phase to a conventional visuomotor learning task.

\section{Estimation of learning rates in the Train phase}


348 We estimated the learning rates in the Train phase with a simple first order state-space model of

349 memory updates to track transitions in reach direction in response to the experienced cursor

350 rotation, as in previous work ${ }^{7}$. In this framework, motor learning is considered a process that

351 estimates perturbations imposed in the task environment. Specifically in the visuomotor rotation $u^{(t)} \quad h^{(t)}$

task, the executed motor plan at trial $t$ determined the direction of the hand movement

$$
h^{(t)}=u^{(t)}
$$

354 While the hand movement was not directly observable for participants, a cursor projected on the

355 screen provided online feedback of the hand motion while the visual rotation $p^{(t)}$ was imposed

356 between the hand movement and the visual cursor:

$$
c^{(t)}=h^{(t)}+p^{(t)}
$$

358 The brain may predict hand movement direction $\hat{h}^{(t)}$ based on the estimation of the perturbation $x^{(t)}$ and the efference copy of the motor plan $u^{(t)}$ :

361 To minimize the sensory prediction error $c^{(t)}-\hat{h}^{(t)}$, the brain updates the estimate of the 362 perturbation with the following learning rule:

$$
\hat{h}^{(t)}=u^{(t)}+x^{(t)} .
$$

364 where the learning rate $\beta$ characterizes the rate of learning. 
365 Here, we estimated the learning rate $\beta$ in the Train phase using the data of hand directions in a

366 triplet of $\mathrm{M}, \mathrm{S}$, and $\mathrm{M}$ trials, following previously developped methods ${ }^{46}$. That is, considering a $\beta^{(k)}$

367 certain trial of $\mathrm{S}$ trials at the trial $t=k, \quad$ was estimated using the measured hand movement

$$
h^{(k)} \quad p^{(k)} \quad h^{(k-1)}
$$

368 direction and the given cursor rotation in S trials at $t=k, \quad$ and the presented

$$
T^{(k-1)} \quad h^{(k+1)} \quad T^{(k+1)}
$$

369 target direction in M trials at $t=k-\frac{1}{T}$, as well as and in M trials at $t=k+$

370 1. Because in $M$ trials, the visual target was presented and the participants were explicitly

371 instructed to cross the target with their unseen but estimated hand position, the participants'

372 estimation of their hand direction should closely match the target direction. We thus assumed $T^{(k-1)}=\hat{h}^{(k-1)} \quad T^{(k+1)}=\hat{h}^{(k+1)} \quad x^{(k-1)}=T^{(k-1)}-h^{(k-1)}$

$$
x^{(k+1)}=T^{(k+1)}-h^{(k+1)} \quad \text {. From (1) and (3), we have and }
$$

. Importantly, because the cursor was not given to participants in M trials $c^{(k-1)}-\hat{h}^{(k-1)}$

at $t=k-1$, the sensory prediction error

$$
x^{(k)}=x^{(k-1)}
$$

was absent and thus, no memory update

376 was engaged. Hence, we assumed

$$
c^{(k)}-\hat{h}^{(k)}
$$

. For subsequent $\mathrm{S}$ trials at $t=k$, because the

Using (4), we estimate $\beta^{(k)}$ by:

$$
\beta^{(k)}=\frac{x^{(k+1)}-x^{(k)}}{c^{(k)}-\hat{h}^{(k)}} .
$$

381 According to (1), (2), (3), and (5), we have:

$$
\beta^{(k)}=\frac{x^{(k+1)}-x^{(k)}}{\left(u^{(k)}+p^{(k)}\right)-\left(u^{(k)}+x^{(k)}\right)} .
$$


383 Then, by applying $x^{(k)}=x^{(k-1)}$, we have:

$$
\beta^{(k)}=\frac{x^{(k+1)}-x^{(k-1)}}{p^{(k)}-x^{(k-1)}} .
$$

385 Finally, by substituting $x^{(k-1)}$ by $T^{(k-1)}-h^{(k-1)}$ and $x^{(k+1)}$ by $T^{(k+1)}-h^{(k+1)}$, we have

$$
\beta^{(k)}=\frac{\left(T^{(k+1)}-h^{(k+1)}\right)-\left(T^{(k-1)}-h^{(k-1)}\right)}{p^{(k)}-\left(T^{(k-1)}-h^{(k-1)}\right)} .
$$

387 Because we measured $T^{(k+1)}-h^{(k+1)}$ and $T^{(k-1)}-h^{(k-1)}$ in M trials and manipulated $p^{(k)}$ as

388 a cursor rotation in S trials, $\beta^{(k)}$ can be estimated for each triplet of M-S-M trials in the Train 389 phase. Note that the denominator $p^{(k)}-\left(T^{(k)}-h^{(k+1)}\right)$ occasionally approaches 0 , resulting in 390 an unreliable estimation of $\beta$. To prevent this, we used the median instead of the mean for each 391 Train phase for each participant before performing statistical analyses.

392 A potential limitation of the above method is that it does not account for the effect of another type of memory that is updated by reward signals ${ }^{24}$, which might be formed in $\mathrm{M}$ trials at $t=k-1$. If

394 this reward-based motor memory exists, it affects the measured reach direction in subsequent $\mathrm{S}$ 395 trials at $t=k$ and M trials at $t=k+1$. This could potentially bias the approximation of the sensory prediction error $p^{(k)}-x^{(k-1)}$ by $p^{(k)}-\left(T^{(k-1)}-h^{(k-1)}\right)$ and thus, bias the estimation of $\beta$.

397 However, according to the theory of reward-based motor memory ${ }^{24}$, the directions of the bias is opposite in the two cases of perturbation sequences: whether the direction of the perturbation switches or remains the same. Because the perturbation sequence was pseudorandomized, this bias should be predominantly eliminated when we calculate the median over each block. If a small effect of this bias remains, it would likely lead to an underestimation of $\beta$ for Lrn groups and 
403 of this potential confounding factor, we examined the generalization of the meta-learning in the

404 Train phase to a conventional visuomotor rotation task where no monetary feedback was given.

\section{Estimation of learning rates in the Generalization phase}

406 Using the state-space model of the memory update (1)-(4), the learning rate was estimated via 407 least square to fit each subject's data of measured hand directions. In the Generalization phase,

408 we assumed that the participants aimed at the presented target $T^{(t)}$ with their prediction of the 409 hand direction $\hat{h}^{(t)}$, so that $\hat{h}^{(t)}$ closely matched with $T^{(t)}$ (i.e, $\hat{h}^{(t)}=T^{(t)}$ ). They observed 410 the error between the cursor and the prediction of the hand $c^{(t)}-\hat{h}^{(t)}$. Thus, the state-space model 411 of the memory update (4) provides us the simulated sequences of $x^{(t)}$ over trials using the 412 measured sequence of $c^{(t)}-T^{(t)}$ for a given $\beta$. The model also generates simulated sequence 413 of participants' reach error $E_{S}{ }^{(t)}=T^{(t)}-h^{(t)}$ in accordance with (1) and (3). In the experiment, 414 we measured the actual participants' reach error $E_{M}{ }^{(t)}=T^{(t)}-h^{(t)}$. Thus, following a previous 415 method $^{4}$, we estimated the learning rate $\beta$ that minimized the sum of the least square error 416 between $E_{S}{ }^{(t)}$ and $E_{M}{ }^{(t)}$ over each step-perturbation sequence of the three Null trials and the 417 five cursor rotation trials.

\section{Experiment groups and score calculations}

419 There were two independent variables, Action-Outcome structure and Valence, respectively, with 420 two levels for each. This resulted in four experiment groups (Figure 1D). The Action-Outcome 421 structure determined how the monetary feedback was computed in four consecutive $\mathrm{M}$ trials $(t=$ $422 k+i, i=\{1,2,3,4\})$ after the $\mathrm{S}$ trial $(t=k)$ both as a function of the memory $x^{k+i}$, measured as 
423 a reaching angle with respect to the target $T^{(k+i)}-h^{(k+i)}$, and as a function of the given

424 perturbation $p^{(k)}$. This regulated whether the memory updates in response to the sensory 425 prediction error $\beta^{(k)}\left(c^{(k)}-\hat{h}^{(k)}\right)$ were encouraged (Lrn) or discouraged (NLrn). Specifically, 426 for Lrn, larger aftereffects of the exposure to the cursor rotation corresponded to larger scores, 427 whereas, for NLrn, smaller aftereffects corresponded to larger scores. In contrast, Valence 428 determined whether monetary feedback was positive (i.e., reward) or negative (i.e., punishment). 429 Therefore, participants could learn more from sensory prediction error to gain more rewards (Lrn430 Rwd) or to avoid larger punishments (Lrn-Pun), allowing them to improve their scores with 431 greater memory updates. Alternatively, they could learn less from sensory prediction error to gain more rewards (NLrn-Rwd) or to avoid larger punishments (Lrn-Pun), allowing them to improve their score with less memory updates.

434 The score ranges for the Rwd and Pun groups were set to $[0,20]$ and $[-20,0]$, respectively. The 435 Lrn-Rwd/Pun group earned the highest score by showing $100 \%$ or more memory for the last 436 experienced rotation in the previous $\mathrm{S}$ trial (i.e., $\frac{T^{(k+1)}-h^{(k+1)}}{p^{(k)}} \geq 1$ in $i^{\text {th }} \mathrm{M}$ trial within the same 437 cycle), and the score was reduced by 1 point for every $10 \%$ less memory until the lowest score 438 (i.e., $-100 \%$ learning) was reached. The Lrn-Rwd/Pun group earned the highest score by showing $4390 \%$ memory, (i.e. $T^{(k+i)}-h^{(k+i)}=0$ ), and the score was reduced by 1 point for every $10 \%$ 440 more/less memory until the lowest score was reached. These were represented as background 441 color patterns in Figure 1D.

\section{Instructions}

443 Before the task, participants were instructed about the experimental flow for the Train phase and 444 the stimuli and feedback in the S and M trials. They were also explicitly informed that the total 445 score would determine their additional monetary compensation and that the task goal was to 
446 maximize their compensation by crossing the target with their hand as closely as possible. In the

447 Rwd conditions, they were told that additional compensation was initially minimum (0 JPY) and

448 accumulated throughout the task. In the Pun condition, participants were told that additional

449 compensation was initially maximum (1,000 JPY) and subtracted throughout the task. In addition,

450 in the S trial, they were told to vary movement directions from trial to trial. This was to avoid the

451 formation of use-dependent behavior ${ }^{43}$. In addition, they were not informed of visual rotation or

452 the relationship between their reach direction and score size to prevent the use of cognitive

453 strategy $^{47}$. Their unawareness of visual rotation throughout the task was confirmed by a written

454 questionnaire after the task was completed asking if they felt a discrepancy between the hand and

455 the cursor during movements.

\section{Statistical analyses}

Removal of target location-dependent bias

458 Due to a gap in height between the physical hand position and the cursor projected on the screen,

459 a bias between the target direction and the reach direction was inevitable. To remove this bias

460 from the analysis, we calculated the mean reach direction for each target location in the Baseline 461 and subtracted from it in the Train phase for all blocks.

463 In Figure 1C, individual mean reach directions with respect to target direction, $T-h$, in the $k$ -

$4641^{\text {th }}$ and $k+1^{\text {th }}$ trials were compared by Wilcoxon paired signed-rank test to examine the aftereffect

465 (i.e., if the reach direction changed after the participants experienced a sensory prediction error in

466 the $k^{\text {th }}$ trial). Because there was no difference across groups in Baseline, all individual data were

467 combined $(n=40)$. The same test was performed for memory updates in the other four blocks on 
468 individual mean differences of $T-h$ in the $k-1^{\text {th }}$ and $k+1^{\text {th }}$ trials across the blocks for each group

469 (Figure 1D, $n=10$ /group).

472 We first used a linear mixed effect model (LMEM) with Action-Outcome structure, Valence, and

473 Block as the fixed effects and Participant as the random intercept effect to examine the change in

$474 \beta$ over the course of the task, as shown in Figure 2A. All interactions between the fixed effects

475 were included. In $\mathrm{R}$ with $1 \mathrm{me} 4$ package ${ }^{48}$, the model was written as:

476 lmer $(\beta \sim$ Action-Outcome $*$ Valence $*$ Block $+(1 \mid$ Participant $))$. In addition, to estimate

477 the marginal slopes of the learning rate change over blocks with respect to Action-Outcome or

478 Valence, we then developed a reduced model without interactions between Action-Outcome and

479 Valence. The model was written as: $\operatorname{lmer}(\beta \sim($ Action-Outcome + Valence $) *$ Block +

480 (1|Participant)).

481 Then, to examine the overall change in learning rate across the task, the change in $\beta$ from Baseline

$482(\Delta \beta)$ was calculated for each block and then averaged across blocks for each participant and fit 483 with the following model: $\operatorname{lm}(\Delta \beta \sim$ Action-Outcome $*$ Valence $)$. Note that because one data 484 point was obtained for each participant, no random effects were included in this model.

To evaluate whether score performance improved, we calculated the total score for each block including Baseline where the score was calculated in the same manner without being presented to the participants, as shown in Figure 2C. Then, the change from Baseline was calculated and averaged across blocks for each participant. The change for each group was analyzed by one- 
sample Wilcoxon signed-rank test with a hypothetical mean of 0 ( $n=10$ /group).

492 The change in learning rate $\Delta \beta$ in the Generalization phase was analyzed similarly to that in the

493 Train phase. Again, individual mean change from Baseline across blocks was calculated by 494 subtracting the Baseline average $\beta$ across Sequence from $\beta$ in the subsequent blocks. Then, $\Delta \beta$ was analyzed with a LMEM, with Action-Outcome structure, Sequence, and their interaction, as

496 fixed effects and Participant as random effect intercepts: lmer $(\Delta \beta \sim$ Action-Outcome *

497 Sequence $+(1 \mid$ Participant $))$. Note that Valence was removed from the model based on the 498 analysis of the change in $\beta$ in the Train phase, which failed to show its significant effect.

499 In addition, to examine time-dependent changes within the Generalization phase more closely, 500 the data were separated into the first and second half of the phase. To examine if the change in each half of the Generalization phase reflected the change in the Train phase, a Pearson correlation analysis was then performed on the changes between the Train and the first/second half of

503 Generalization phases. Finally, $\Delta \beta$ from each half were analyzed with a linear model with Action-

504 Outcome as the only factor.

506 All data processing and statistical analyses were performed in R version 3.5.1 using the $1 \mathrm{~m}, 1 \mathrm{me} 4^{48}$, 507 lmerTest $^{49}$, and emmeans ${ }^{50}$ packages. For the linear model analyses, effect coding was used to 508 represent the categorical variables (i.e., Action-Outcome and Valence), except in estimation of 509 marginal slopes where dummy coding was used, and the numerical variables (i.e., Block and 510 Sequence) were represented in z-score (i.e., centered and normalized). Lrn and Rwd were set as 511 reference in coding (i.e., negative value in effect coding and zero in dummy coding). The 

a model that treats them as continuous, and (2) confirming the normality of residuals by ShapiroWilk test.

\section{References}

5181 Hattie, J., Biggs, J. \& Purdie, N. Effects of learning skills interventions on student 519 learning: A meta-analysis. Rev Educ Res 66, 99-136, doi:Doi 10.3102/00346543066002099 (1996).

2 Brown, A. L. \& Kane, M. J. Preschool children can learn to transfer: learning to learn and learning from example. Cogn Psychol 20, 493-523 (1988).

3 Harlow, H. F. Physiological psychology. Annu Rev Physiol 11, 269-296, doi:10.1146/annurev.ph.11.030149.001413 (1949).

4 Smith, M. A., Ghazizadeh, A. \& Shadmehr, R. Interacting adaptive processes with different timescales underlie short-term motor learning. PLoS Biol 4, e179, doi:10.1371/journal.pbio.0040179 (2006).

5 Leow, L. A., Loftus, A. M. \& Hammond, G. R. Impaired savings despite intact initial learning of motor adaptation in Parkinson's disease. Exp Brain Res 218, 295-304, doi:10.1007/s00221-012-3060-5 (2012).

6 Kojima, Y., Iwamoto, Y. \& Yoshida, K. Memory of learning facilitates saccadic adaptation in the monkey. $J$ Neurosci 24, 7531-7539, doi:10.1523/JNEUROSCI.174104.2004 (2004).

7 Herzfeld, D. J., Vaswani, P. A., Marko, M. K. \& Shadmehr, R. A memory of errors in sensorimotor learning. Science 345, 1349-1353, doi:10.1126/science.1253138 (2014).

8 Krakauer, J. W., Ghilardi, M. F. \& Ghez, C. Independent learning of internal models for kinematic and dynamic control of reaching. Nat Neurosci 2, 1026-1031, doi:10.1038/14826 (1999).

9 Medina, J. F., Garcia, K. S. \& Mauk, M. D. A mechanism for savings in the cerebellum. J Neurosci 21, 4081-4089 (2001).

10 Tseng, Y. W., Diedrichsen, J., Krakauer, J. W., Shadmehr, R. \& Bastian, A. J. Sensory prediction errors drive cerebellum-dependent adaptation of reaching. J Neurophysiol 98 , 54-62, doi:10.1152/jn.00266.2007 (2007).

11 Wolpert, D. M., Doya, K. \& Kawato, M. A unifying computational framework for motor control and social interaction. Philos Trans R Soc Lond B Biol Sci 358, 593-602, doi:10.1098/rstb.2002.1238 (2003).

12 Cullen, K. E. \& Brooks, J. X. Neural correlates of sensory prediction errors in monkeys: evidence for internal models of voluntary self-motion in the cerebellum. Cerebellum 14, 31-34, doi:10.1007/s12311-014-0608-x (2015).

13 Galea, J. M., Mallia, E., Rothwell, J. \& Diedrichsen, J. The dissociable effects of punishment and reward on motor learning. Nat Neurosci 18, 597-602, doi:10.1038/nn.3956 (2015).

14 Nikooyan, A. A. \& Ahmed, A. A. Reward feedback accelerates motor learning. $J$ Neurophysiol 113, 633-646, doi:10.1152/jn.00032.2014 (2015).

556

15 van der Kooij, K. \& Overvliet, K. E. Rewarding imperfect motor performance reduces adaptive changes. Exp Brain Res 234, 1441-1450, doi:10.1007/s00221-015-4540-1 
(2016).

16 Song, Y. \& Smiley-Oyen, A. L. Probability differently modulating the effects of reward and punishment on visuomotor adaptation. Exp Brain Res 235, 3605-3618, doi:10.1007/s00221-017-5082-5 (2017).

17 Quattrocchi, G., Greenwood, R., Rothwell, J. C., Galea, J. M. \& Bestmann, S. Reward and punishment enhance motor adaptation in stroke. J Neurol Neurosurg Psychiatry 88, 730-736, doi:10.1136/jnnp-2016-314728 (2017).

18 Guitart-Masip, M. et al. Go and no-go learning in reward and punishment: interactions between affect and effect. Neuroimage 62, 154-166, doi:10.1016/j.neuroimage.2012.04.024 (2012).

19 Kojima, Y. \& Soetedjo, R. Selective reward affects the rate of saccade adaptation. Neuroscience 355, 113-125, doi:10.1016/j.neuroscience.2017.04.048 (2017).

20 Izawa, J., Rane, T., Donchin, O. \& Shadmehr, R. Motor adaptation as a process of reoptimization. $J$ Neurosci 28, 2883-2891, doi:10.1523/JNEUROSCI.5359-07.2008 (2008).

21 Therrien, A. S., Wolpert, D. M. \& Bastian, A. J. Effective reinforcement learning following cerebellar damage requires a balance between exploration and motor noise. Brain 139, 101-114, doi:10.1093/brain/awv329 (2016).

22 Pekny, S. E., Izawa, J. \& Shadmehr, R. Reward-dependent modulation of movement variability. J Neurosci 35, 4015-4024, doi:10.1523/JNEUROSCI.3244-14.2015 (2015).

23 Manley, H., Dayan, P. \& Diedrichsen, J. When money is not enough: awareness, success, and variability in motor learning. PLoS One 9, e86580, doi:10.1371/journal.pone.0086580 (2014).

24 Izawa, J. \& Shadmehr, R. Learning from sensory and reward prediction errors during motor adaptation. PLoS Comput Biol 7, e1002012, doi:10.1371/journal.pcbi.1002012 (2011).

25 Finn, C., Xu, K. \& Levine, S. Probabilistic Model-Agnostic Meta-Learning. Adv Neur In 31 (2018).

26 Duan, Y. et al. R1 $\$^{\wedge} 2 \$$ : Fast reinforcement learning via slow reinforcement learning. arXiv preprint arXiv:1611.02779 (2016).

27 Wang, J. X. et al. Prefrontal cortex as a meta-reinforcement learning system. Nat Neurosci 21, 860-868, doi:10.1038/s41593-018-0147-8 (2018).

28 Sutton, R. S. \& Barto, A. G. Reinforcement learning : an introduction. Second edition. edn, (The MIT Press, 2018).

29 Izawa, J., Criscimagna-Hemminger, S. E. \& Shadmehr, R. Cerebellar contributions to reach adaptation and learning sensory consequences of action. J Neurosci 32, 4230-4239, doi:10.1523/JNEUROSCI.6353-11.2012 (2012).

30 Ishii, S., Yoshida, W. \& Yoshimoto, J. Control of exploitation-exploration metaparameter in reinforcement learning. Neural Netw 15, 665-687 (2002).

31 Doya, K. Metalearning and neuromodulation. Neural Netw 15, 495-506 (2002).

32 Schweighofer, N. \& Doya, K. Meta-learning in reinforcement learning. Neural Netw 16, 5-9 (2003).

33 Gonzalez Castro, L. N., Hadjiosif, A. M., Hemphill, M. A. \& Smith, M. A. Environmental consistency determines the rate of motor adaptation. Curr Biol 24, 1050-1061, doi:10.1016/j.cub.2014.03.049 (2014).

34 Hajcak, G. \& Foti, D. Errors are aversive: defensive motivation and the error-related negativity. Psychol Sci 19, 103-108, doi:10.1111/j.1467-9280.2008.02053.x (2008).

35 Torrecillos, F., Albouy, P., Brochier, T. \& Malfait, N. Does the processing of sensory and reward-prediction errors involve common neural resources? Evidence from a frontocentral negative potential modulated by movement execution errors. $J$ Neurosci 34 , 4845-4856, doi:10.1523/JNEUROSCI.4390-13.2014 (2014). 
$60836 \quad$ Kawato, M. \& Wolpert, D. Internal models for motor control. Novartis Found Symp 218, 609 291-304; discussion 304-297 (1998).

61037 Mathis, M. W., Mathis, A. \& Uchida, N. Somatosensory Cortex Plays an Essential Role 611 in Forelimb Motor Adaptation in Mice. Neuron 93, 1493-1503 e1496, doi:10.1016/j.neuron.2017.02.049 (2017).

614

38 Wolpert, D. M., Miall, R. C. \& Kawato, M. Internal models in the cerebellum. Trends Cogn Sci 2, 338-347 (1998).

615

39 Ishikawa, T., Tomatsu, S., Izawa, J. \& Kakei, S. The cerebro-cerebellum: Could it be loci of forward models? Neurosci Res 104, 72-79, doi:10.1016/j.neures.2015.12.003 (2016).

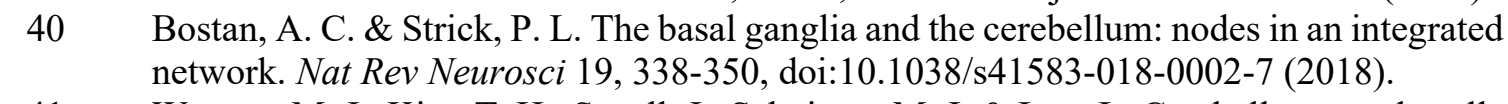

41 Wagner, M. J., Kim, T. H., Savall, J., Schnitzer, M. J. \& Luo, L. Cerebellar granule cells encode the expectation of reward. Nature 544, 96-100, doi:10.1038/nature21726 (2017).

42 Kostadinov, D., Beau, M., Pozo, M. B. \& Hausser, M. Predictive and reactive reward signals conveyed by climbing fiber inputs to cerebellar Purkinje cells. Nat Neurosci, doi:10.1038/s41593-019-0381-8 (2019).

43 Verstynen, T. \& Sabes, P. N. How each movement changes the next: an experimental and theoretical study of fast adaptive priors in reaching. J Neurosci 31, 10050-10059, doi:10.1523/JNEUROSCI.6525-10.2011 (2011).

44 Schaefer, S. Y., Shelly, I. L. \& Thoroughman, K. A. Beside the point: motor adaptation without feedback-based error correction in task-irrelevant conditions. J Neurophysiol 107, 1247-1256, doi:10.1152/jn.00273.2011 (2012).

45 Neville, K. M. \& Cressman, E. K. The influence of awareness on explicit and implicit contributions to visuomotor adaptation over time. Exp Brain Res 236, 2047-2059, doi:10.1007/s00221-018-5282-7 (2018).

46 Marko, M. K., Haith, A. M., Harran, M. D. \& Shadmehr, R. Sensitivity to prediction error in reach adaptation. J Neurophysiol 108, 1752-1763, doi:10.1152/jn.00177.2012 (2012).

47 Taylor, J. A., Klemfuss, N. M. \& Ivry, R. B. An explicit strategy prevails when the cerebellum fails to compute movement errors. Cerebellum 9, 580-586, doi:10.1007/s12311-010-0201-x (2010).

48 Bates, D., Machler, M., Bolker, B. M. \& Walker, S. C. Fitting Linear Mixed-Effects Models Using lme4. J Stat Softw 67, 1-48 (2015).

49 Kuznetsova, A., Brockhoff, P. B. \& Christensen, R. H. B. lmerTest Package: Tests in Linear Mixed Effects Models. J Stat Softw 82, 1-26 (2017).

50 Estimated Marginal Means, aka Least-Squares Means (https://CRAN.Rproject.org/package=emmeans, 2019). 